\title{
UMA PROPOSTA CONCEITUAL PARA A MEDIDA DO GRAU DE COMPETITIVIDADE DE UMA EMPRESA
}

\section{A CONCEPTUAL PROPOSAL TO MEASURE THE COMPETITIVENESS DEGREE OF A COMPANY}

\author{
Wagner Cezar Lucato, M.Sc \\ Doutorando em Engenharia de Produção \\ UNIMEP - Universidade Metodista de Piracicaba \\ Programa de Pós-Graduação em Engenharia de Produção \\ Rod. Santa Bárbara - Iracemápolis, KM 01 \\ CEP 13450-000 Santa Bárbara D’Oeste - $\underline{\text { SP }}$
}

Telefone: (11) 4438-1803 - e-mail: wlucato@terra.com.br

\section{Prof. Dr. Milton Vieira Júnior}

Professor Associado

UNIMEP - Universidade Metodista de Piracicaba

Programa de Pós-Graduação em Engenharia de Produção

Rod. Santa Bárbara - Iracemápolis, KM 01

CEP 13450-000 Santa Bárbara D’Oeste - $\underline{\text { SP }}$

Telefone: (19) 3124-1769 - e-mail: mvieira@unimep.br

\section{RESUMO}

Este trabalho tem como objetivo propor uma estrutura teórica para a avaliação do grau de competitividade de empresas que atuem no mesmo segmento industrial. Adotando como pressupostos a definição de competitividade empresarial e os conceitos de variáveis competitivas e de perfil competitivo, o presente estudo vai buscar na Análise dos Agrupamentos a fundamentação teórica para propor o conceito de distância competitiva, como a grandeza para medir o grau de competitividade que se pretende determinar. Uma exemplificação prática, ilustrando a aplicação dos conceitos propostos a empresas brasileiras do ramo de autopeças, completa a proposição ora apresentada.

Palavras-chave: competitividade, perfil competitivo, distância competitiva. 


\begin{abstract}
The main objective of this paper is to present a conceptual proposal to measure the competitiveness degree of companies from the same industry. It starts by establishing as assumptions the definition of enterprise competitiveness and the concepts of competitive variables and competitive profile. To measure the competitiveness degree of a company, this study proposes the concept of competitive distance, which can verify how similar a competitive profile is as compared to a standard. A practical example considereing companies from the Brazilian automobile part supplier industry is presented to show the feasibility of using the proposed concepts.
\end{abstract}

Key-words: competitiveness, competitive profile, competitive distance.

\title{
1. INTRODUÇÃO
}

Este trabalho tem como objetivo propor uma forma de medir o grau de competitividade de uma empresa. Porém, a competitividade empresarial é um fenômeno bastante complexo e para poder medi-la será necessário antes estabelecer algumas definições básicas para suportar o desenvolvimento que aqui se propõe. Tais conceitos, no entanto, serão admitidos como pressupostos para este trabalho, uma vez que a discussão de sua fundamentação transcende o objetivo da proposição que aqui se apresenta.

Assim, assumem-se no presente desenvolvimento três definições basilares para a proposição da medida do grau de competitividade de uma empresa: o que se considerará como competitividade empresarial, como variáveis competitivas e como perfil competitivo de uma empresa.

Para o conceito de competitividade empresarial, propõe-se adotar, como um pressuposto, uma definição que integre múltiplos aspectos sob diferentes enfoques: o mercadológico, o estratégico, o de produção, o de finanças, o da mobilização de pessoas, o do meio ambiente, o da integração aos mercados globais e o da conectividade à Internet. Dessa maneira, a proposta de uma forma de medir a competitividade empresarial irá considerar que:

A competitividade de uma empresa é a sua capacidade de estar, continuamente, criando e renovando suas estratégias concorrenciais (HAMEL \& PRAHALAD, 1995), como forma de obter um posicionamento favorável no mercado (KOTLER \& ARMSTRONG, 2002). Como resultado, deve gerar uma rentabilidade acima da média do setor industrial onde atua (PORTER, 1989), operando de maneira financeiramente sustentável (ROSS et al., 2002), com qualidade, rapidez e flexibilidade (SLACK et al., 2002). Ainda, deve estar alinhada com 


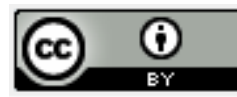

o mercado globalizado (DANIELS \& DANIELS, 1994), integrada à Internet (CASTELLS, 2000) e em perfeita harmonia com seus colaboradores (PFEFFER, 1996) e com seu meio ambiente (PORTER \& LINDE, 1995).

Para ser possível a mediada do grau de competitividade de uma empresa também será necessário traduzir todas as características competitivas enunciadas na definição acima na forma de variáveis, que serão denominadas variáveis competitivas. Para ilustrar o desenvolvimento que se pretende e com base em LUCATO (2003), assume-se também como pressuposto para este trabalho um conjunto de seis variáveis quantitativas e seis variáveis qualitativas do tipo dicotômicas que têm como finalidade caracterizar o desempenho competitivo de uma empresa em sua atuação no mercado. Um sumário das variáveis competitivas consideradas como exemplo para uma melhor compreensão deste trabalho vai mostrado no Quadro 1, abaixo.

Quadro 1. Variáveis competitivas consideradas como exemplos.

\begin{tabular}{|l|l|l|l|}
\hline \multicolumn{1}{|c|}{ Enfoque } & \multicolumn{1}{|c|}{ Variável Competitiva } & \multicolumn{1}{c|}{ Como Medir } & \multicolumn{1}{c|}{$\begin{array}{c}\text { Tipo de } \\
\text { Variável }\end{array}$} \\
\hline Mercadológico & $\begin{array}{l}\text { Posicionamento favorável de } \\
\text { mercado, com crescimento de } \\
\text { vendas }\end{array}$ & $\begin{array}{l}\text { Participação mercado } \\
\text { Crescimento de vendas }\end{array}$ & $\begin{array}{l}\text { Quantitativa } \\
\text { Quantitativa }\end{array}$ \\
\hline Estratégico & $\begin{array}{l}\text { Eficácia na geração e renovação de } \\
\text { suas estratégias }\end{array}$ & $\begin{array}{l}\text { Rentabilidade (ROE) } \\
\text { Crescimento de vendas }\end{array}$ & $\begin{array}{l}\text { Quantitativa } \\
\text { Quantitativa }\end{array}$ \\
\hline Produção & $\begin{array}{l}\text { Existência de Sistema de Qualidade } \\
\text { Filosofia Just-in-Time de produção }\end{array}$ & Certificação ISO 9000 & Adoção de técnicas JIT \\
\hline Finanças & $\begin{array}{l}\text { Rentabilidade acima da média } \\
\text { Boa liquidez a curto prazo } \\
\text { Boa liquidez a longo prazo } \\
\text { Baixo endividamento }\end{array}$ & $\begin{array}{l}\text { Rentabilidade (ROE) } \\
\text { Índice liquidez corrente } \\
\text { Índice de liquidez geral } \\
\text { Índice de endividamento geral }\end{array}$ & Dicotômica \\
\hline $\begin{array}{l}\text { Mobilização de } \\
\text { Pessoas }\end{array}$ & Princípios de gestão de R.H. & $\begin{array}{l}\text { Prática de 5 princípios da moderna } \\
\text { gestão de RH }\end{array}$ & $\begin{array}{l}\text { Quantitativa } \\
\text { Quantitativa } \\
\text { Quantitativa }\end{array}$ \\
\hline Ambiental & $\begin{array}{l}\text { Existência de um Sistema de Gestão } \\
\text { Ambiental (SGA) }\end{array}$ & $\begin{array}{l}\text { SGA estabelecido conforme ISO } \\
\text { 14000 }\end{array}$ & Dicotômica \\
\hline $\begin{array}{l}\text { Integração aos } \\
\text { Mercados } \\
\text { Globais }\end{array}$ & $\begin{array}{l}\text { Participação evolutiva no mercado } \\
\text { global }\end{array}$ & $\begin{array}{l}\text { Atuação ativa no comércio } \\
\text { internacional }\end{array}$ \\
\hline Conectividade & $\begin{array}{l}\text { Estar efetivamente integrada à } \\
\text { Internet }\end{array}$ & Ter um site de, pelo menos, 3 geração & Dicotômica \\
\hline
\end{tabular}

Fonte: Lucato (2003).

Assim, como ilustra o quadro acima, em função do conceito de competitividade e de suas respectivas variáveis, este trabalho propõe construir uma estrutura teórica de avaliação do grau de competitividade de uma empresa considerando, como exemplo ilustrativo, as seguintes variáveis competitivas (LUCATO, 2003): 
a) seis variáveis quantitativas :

(1) a participação de mercado, (2) o crescimento de vendas, (3) o retorno sobre o patrimônio líquido, (4) o índice de liquidez corrente, (5) o índice de liquidez geral e (6) o índice de endividamento geral;.

b) seis variáveis qualitativas nominais do tipo dicotômicas :

(1) a existência efetiva de um sistema de qualidade certificado conforme as normas ISO 9000, (2) a utilização de técnicas just-in-time na produção, (3) a prática efetiva de, pelo menos, 5 princípios básicos da gestão moderna de recursos humanos, (4) a existência efetiva de um sistema de gestão ambiental em linha com as exigências da norma ISO 14001, (5) uma atuação permanente, estruturada e em constante evolução no comércio internacional e (6) possuir um site na Internet que seja, pelo menos, de terceira geração (transações on-line).

Como resultado dessa estrutura conceitual, este trabalho assume, como um terceiro pressuposto, a definição de perfil competitivo de uma empresa, como sendo o conjunto dos valores assumidos por todas essas variáveis competitivas, em um determinado período de tempo. Como exemplo, uma empresa " $\mathrm{X}$ " poderia ter o seu perfil competitivo definido por:

$$
\mathrm{PC}_{\mathrm{X}}=\{20,5 \% ; 10,2 \% ; 22,0 \% ; 1,25 ; 0,81 ; 0,79 ; 1 ; 0 ; 0 ; 1 ; 1 ; 1\}
$$

onde cada elemento desse conjunto é representado pelo valor da respectiva variável competitiva, expresso na mesma ordem enunciada acima. Para as variáveis dicotômicas, o número (1) é associado à presença de determinada característica e o (0) à não presença.

Definida a competitividade empresarial e determinadas as variáveis que irão caracterizá-la, pode-se partir para o estabelecimento de uma forma de medi-la, como irá se propor a seguir.

\section{A PROPOSTA DE UMA CONCEPÇÃO TEÓRICA}

O desenvolvimento de uma grandeza que permita determinar o grau de competitividade de uma empresa deve partir do estabelecimento de uma forma de medi-lo. Ferreira (1999, p.1.110) explica que medir significa "determinar ou verificar, tendo por base 
uma escala fixa, a extensão, medida ou grandeza de". Assim, o ato de medir implica na existência de um padrão ou referência contra o qual a grandeza a ser avaliada é comparada.

Verifica-se, portanto, que, para se medir o grau de competitividade de uma empresa, haverá a necessidade de se comparar o seu perfil competitivo com um perfil competitivo padrão a ser estabelecido. Dessa forma, a questão que se coloca, de início, é a necessidade da definição de um referencial de competitividade, que passará a ser o paradigma competitivo contra o qual comparar-se-á o perfil da empresa cuja competitividade se deseja determinar.

Para essa finalidade, propõe-se estabelecer como padrão de comparação o perfil competitivo de uma empresa que, segundo algum critério objetivo, seja considerada o padrão competitivo do mesmo segmento industrial ao qual pertença a empresa cujo grau de competitividade se deseja medir. Existem várias alternativas para o critério a ser adotado na seleção desse referencial competitivo: (a) eleger o líder do mercado, (b) considerar um concorrente específico que seja considerado modelo de competitividade por sua destacada atuação no segmento industrial, (benchmarking), (c) assumir uma média entre as empresas que detenham as maiores participações de mercado no segmento industrial em análise e (d) outro critério que atenda aos interesses do pesquisador.

A utilização dessa metodologia para o cálculo do grau de competitividade de uma empresa gerará dois conjuntos de variáveis competitivas: (1) o perfil competitivo da empresa cujo grau de competitividade se deseja medir e que a partir de agora será chamada de empresa em estudo ou em análise, e (2) o perfil competitivo da empresa escolhida como padrão.

Medir o grau de competitividade da empresa em análise consistirá em se determinar uma grandeza que defina a proximidade, ou o afastamento, que seu perfil competitivo tenha em relação ao perfil competitivo padrão. Essa grandeza será designada por distância competitiva e será a base do desenvolvimento sugerido por este trabalho.

Segundo essa proposta, a distância competitiva será a grandeza que permitirá medir o grau de competitividade de uma empresa, em relação a um padrão. Como consequiência, possibilitará também saber, entre duas empresas, qual a mais competitiva dentre elas. Para tanto, será suficiente a determinação da distância competitiva nos dois casos. A mais competitiva será a que mais se aproxima, ou menos se afasta, de um padrão comum utilizado como base para essa comparação.

Estabelecida conceitualmente a forma de medir o grau de competitividade de uma empresa, o problema que resta é como definir objetivamente a forma de se calcular a distância competitiva como aqui sugerida. Uma proposição para essa determinação é mostrada a seguir. 


\section{A DETERMINAÇÃO DA DISTNCIA COMPETITIVA}

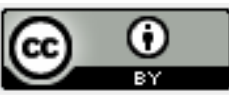

Do que se apresentou no item anterior, pode-se, de maneira bastante sucinta, afirmar que o cálculo da distância competitiva envolve a escolha de um critério que permita qualificar quanto o perfil competitivo de uma empresa é parecido, ou diferente, de um referencial estabelecido. A escolha de um critério que meça a distância entre dois objetos, ou que quantifique quanto eles são parecidos, é tema de um capítulo da Matemática que se denomina Análise de Agrupamentos. É nesse ambiente teórico que este trabalho irá buscar os fundamentos para o cálculo da distância competitiva.

Segundo Bussab et al. (1990, p.16), pode-se verificar quanto dois objetos são parecidos, ou diferentes, através de uma medida que se denomina "coeficiente de parecença". Essa grandeza permite indicar as similaridades ou dissimilaridades entre os valores observados. Os autores ainda afirmam que o coeficiente de parecença pode ser de dois tipos: (1) medidas de similaridade e (2) medidas de dissimilaridade. No primeiro caso, quanto maior o valor observado, mais parecidos serão os objetos. No segundo, quanto maior o valor observado, menos parecidos (mais dissimilares) serão os objetos.

Como exemplos de coeficientes de parecença, pode-se citar o coeficiente de correlação como sendo uma medida de similaridade, já que, quanto maior for o esse coeficiente entre dois grupos de dados, mais parecidos eles serão. A distância euclidiana, por sua vez, é uma medida de dissimilaridade, pois quanto maior for a distância euclidiana entre dois objetos, mais dissimilares eles serão. Essa distinção, no entanto, não é fundamental, na medida em que, de maneira geral, é possível estabelecer uma medida de dissimilaridade a partir de outra de similaridade e vice-versa.

Com base nesse referencial teórico, propõe-se estabelecer um coeficiente de parecença que mostre as dissimilaridades entre os perfis competitivos de duas empresas: a empresa A, considerada como referencial competitivo, e a empresa B, aquela cuja competitividade se deseja medir e que será analisada comparativamente ao padrão estabelecido. Para isso, e levando em conta as variáveis competitivas, a determinação do coeficiente de parecença pretendido recomenda considerar separadamente os dois tipos de variáveis que o perfil competitivo proposto por este trabalho envolve: as variáveis quantitativas e as variáveis qualitativas nominais do tipo dicotômicas. 
Segundo Bussab et al. (1990, p.16), para as variáveis quantitativas, pode-se utilizar como coeficiente de parecença a "distância euclidiana simples" entre um objeto A (empresa tomada como referência) e outro B (empresa em estudo) e que pode ser assim determinada:

$$
d_{e}(A, B)=\left[\sum\left(\left(x_{i}(A)-x_{i}(B)\right)^{2}\right]\right.
$$

(Relação 1)

na qual: $\quad d_{e}(A, B)$ - é a distância euclidiana entre a empresa A (referência) e a empresa

B (caso em estudo);

$\mathrm{X}_{\mathrm{i}}(\mathrm{A})$ - é a variável i da empresa A (referência)

$\mathrm{x}_{\mathrm{i}}$ (B) - é a variável i da empresa $\mathrm{B}$ (caso em estudo)

Como mostra a definição acima, o que a distância euclidiana procura determinar é quanto o perfil competitivo da empresa A difere do perfil competitivo da empresa B, levandose em conta apenas as variáveis quantitativas. Assim, aplicando-se esse conceito às vaiáveis competitivas consideradas por este trabalho, a relação acima assume o seguinte aspecto:

Relação (2)

$$
\begin{array}{r}
d_{e}(A, B)=\left[\left(X_{1}(A)-X_{1}(B)\right)^{2}+\left(X_{2}(A)-X_{2}(B)\right)^{2}+\left(X_{3}(A)-X_{3}(B)\right)^{2}+\right. \\
\left(X_{4}(A)-X_{4}(B)\right)^{2}+\left(X_{5}(A)-X_{5}(B)\right)^{2}+\left(X_{6}(A)-X_{6}(B)\right)^{2}
\end{array}
$$

Nessa relação: $d_{e}(A, B) \quad$ - distância euclidiana entre o perfil competitivo da empresa A (referência) e da empresa B (empresa em estudo)

$\mathrm{X}_{1}(\mathrm{~A}) \quad$ - participação de mercado da empresa $\mathrm{A}$

$\mathrm{X}_{1}(\mathrm{~B}) \quad$ - participação de mercado da empresa B

$\mathrm{X}_{2}(\mathrm{~A}) \quad$ - crescimento de vendas da empresa $\mathrm{A}$

$\mathrm{X}_{2}(\mathrm{~B}) \quad$ - crescimento de vendas da empresa B

$\mathrm{X}_{3}(\mathrm{~A}) \quad$ - retorno sobre o patrimônio líquido da empresa $\mathrm{A}$

$\mathrm{X}_{3}(\mathrm{~B}) \quad$ - retorno sobre o patrimônio líquido da empresa $\mathrm{B}$

$\mathrm{X}_{4}(\mathrm{~A}) \quad$ - índice de liquidez corrente da empresa $\mathrm{A}$

$\mathrm{X}_{4}(\mathrm{~B}) \quad$ - índice de liquidez corrente da empresa B

$\mathrm{X}_{5}(\mathrm{~A}) \quad$ - índice de liquidez geral da empresa $\mathrm{A}$ 


$$
\begin{array}{ll}
\mathrm{X}_{5}(\mathrm{~B}) & \text { - índice de liquidez geral da empresa } \mathrm{B} \\
\mathrm{X}_{6}(\mathrm{~A}) & \text { - índice de endividamento da empresa } \mathrm{A} \\
\mathrm{X}_{6}(\mathrm{~B}) & \text { - índice de endividamento da empresa B }
\end{array}
$$

Porém, as variáveis quantitativas consideradas neste trabalho envolvem grandezas não comparáveis diretamente. A participação de mercado, o crescimento de vendas e o retorno sobre o patrimônio líquido são expressos em porcentagem. Já os índices são números puros. Como essas grandezas precisam ser somadas para a determinação da distância euclidiana, a sua consideração, na forma em que se apresentam, pode induzir a resultados de difícil interpretação. Para contornar esse problema, Bussab et al. (1990, p.18) recomendam a utilização de "variáveis padronizadas", que são bastante utilizadas na Estatística. Essa prática considera que todas as variáveis empregadas são reduzidas a números puros, eliminando-se dessa maneira as diferentes unidades de medida para cada variável estudada. Conforme sugere Costa Neto (1977, p.238), uma variável padronizada pode ser obtida através da utilização da transformação:

$$
Z_{i}=\frac{x_{i}(.)-\overline{X_{i}}}{s_{i}}
$$

(Relação 3)

na qual $\overline{\mathrm{x}}_{\mathrm{i}}$ e $\mathrm{s}_{\mathrm{i}}$ indicam, respectivamente, a média e o desvio padrão da i-ésima variável.

Feita a transformação, a distância euclidiana passa então a ser expressa por :

$$
\mathrm{d}_{\mathrm{e}}(\mathrm{A}, \mathrm{B}) \stackrel{1 / 2}{=}\left[\sum\left(\left(\mathrm{z}_{\mathrm{i}}(\mathrm{A})-\mathrm{z}_{\mathrm{i}}(\mathrm{B})\right)^{2}\right]\right.
$$

Assim, o cálculo da distância euclidiana se processará de maneira análoga à proposta pela Relação (1) acima, com a diferença que as variáveis utilizadas estarão em sua forma padronizada. Comentário equivalente pode ser feito quanto ao cálculo da distância euclidiana entre o perfil competitivo da empresa padrão (A) e o perfil competitivo da empresa em estudo (B). A Relação (2) acima poderá ser aplicada da mesma forma como proposta, desde que se tome o cuidado de utilizar para cada variável $\mathrm{X}_{\mathrm{i}}$ o seu valor padronizado, cujo cálculo é feito conforme a Relação (3) já descrita. 
Até o presente momento, o cálculo da distância euclidiana simples só levou em conta as variáveis quantitativas. Porém, o perfil competitivo de uma empresa, segundo as considerações deste trabalho, envolve também variáveis qualitativas nominais do tipo dicotômicas. Será necessário então desenvolver um critério que permita avaliar as similaridades, ou dissimilaridades, entre os perfis competitivos da empresa padrão e da empresa em estudo, considerando-se agora as variáveis qualitativas. Para tanto, a abordagem teórica deverá seguir os mesmos princípios conceituais já estabelecidos anteriormente, porém adaptados às peculiaridades das variáveis dicotômicas. Mais uma vez recorre-se aos ensinamentos da Análise de Agrupamentos. Bussab et al. (1990, p.24) sugerem como coeficiente de parecença, para as variáveis qualitativas nominais do tipo dicotômicas, a utilização da distância euclidiana média, estabelecida conforme ilustra o exemplo do Quadro 2 a seguir. Nesse exemplo, procura-se analisar a semelhança, ou diferença, entre duas cidades A e B, em função da disponibilidade de determinados serviços assistenciais. Para a presença de determinado serviço será atribuída a designação (1) e a não presença receberá a indicação (0). A similaridade, ou dissimilaridade, entre as duas cidades será definida por uma grandeza denominada distância euclidiana média, que é conhecida na literatura como "distância binária de Sokal" e calculada conforme o exemplo do Quadro 2 a seguir. Como a comparação entre os perfis competitivos das empresas em estudo e padrão assemelha-se ao exemplo descrito, propõe-se utilizar neste trabalho como coeficiente de parecença, para as variáveis dicotômicas, a distância binária de Sokal, calculada entre as variáveis competitivas dicotômicas da empresa A (referência) e as variáveis competitivas dicotômicas da empresa B (caso em estudo), como segue:

$$
d_{b}(A, B)=\left[\begin{array}{c}
b+c \\
-----\cdot c+d
\end{array}\right]^{1 / 2}
$$

onde: $d_{b}(A, B)$ - distância binária de Sokal entre as empresas A (padrão) e B (em estudo).
a - número de ocorrências nas quais as empresas A e $\mathrm{B}$ possuem um atributo.
b - número de ocorrências nas quais A não possui um atributo mas a B o possui.
c - número de ocorrências nas quais A possui um atributo mas B não o possui
d - número de ocorrências nas quais nem A e a nem B possuem um atributo. 
Quadro 2. Definição e cálculo da distância binária de Sokal

Resultados da presença (1) ou ausência (0) de serviços assistenciais (X) em duas cidades A e B:

$\begin{array}{llllllll}\text { Variável } & \mathrm{X} 1 & \mathrm{X} 2 & \mathrm{X} 3 & \mathrm{X} 4 & \mathrm{X} 5 & \mathrm{X} 6 & \mathrm{X} 7 \\ \text { Cidade A } & 1 & 1 & 0 & 1 & 0 & 1 & 0 \\ \text { Cidade B } & 1 & 0 & 0 & 1 & 1 & 0 & 1\end{array}$

Número observado de pares $(1,1),(1,0),(0,1)$ e $(0,0)$ :

\begin{tabular}{|c|ccc|c|}
\cline { 2 - 4 } \multicolumn{1}{c|}{} & \multicolumn{1}{c}{1} & 0 & Total \\
\cline { 2 - 5 } Cidade B & $\mathrm{a}=2$ & $\mathrm{~b}=2$ & $\mathrm{a}+\mathrm{b}=4$ \\
0 & $\mathrm{c}=2$ & $\mathrm{~d}=1$ & $\mathrm{c}+\mathrm{d}=3$ \\
\hline & Total & $\mathrm{a}+\mathrm{c}=4$ & $\mathrm{~b}+\mathrm{d}=3$ & $\mathrm{a}+\mathrm{b}+\mathrm{c}+\mathrm{d}=7$ \\
\cline { 2 - 5 } & &
\end{tabular}

A distância euclideana média, neste caso, recebe a denominação de "distância binária de Sokal" e pode ser calculada da seguinte forma :

$$
d_{b}(A, B)=\left[\begin{array}{c}
b+c \\
-a+b+c+d
\end{array}\right]^{1 / 2}
$$

Ela indica a proporção de atributos não coincidentes nos dois objetos em estudo.

Quanto maior esse número, mais diferentes serão os objetos, tratando-se portanto de uma medida de dissimilaridade. No exemplo acima:

$$
d_{b}(A, B)=\left[\begin{array}{c}
2+2 \\
------ \\
7
\end{array}\right]^{1 / 2}=0,76
$$

Fonte: adaptado de BUSSAB et al. (1990, p. 25)

Até agora se propôs a utilização de dois critérios distintos para se medir a similaridade, ou dissimilaridade, dos perfis competitivos da empresa padrão e da empresa em estudo: (1) a distância euclidiana simples para as variáveis quantitativas e (2) a distância binária de Sokal para as variáveis qualitativas nominais do tipo dicotômicas. 
Porém, há a necessidade de se compor essas duas grandezas de modo a poder considerá-las de maneira conjunta. Romesburg citado por Bussab et al. (1990, p.31), sugere a elaboração de um "coeficiente combinado de semelhança", no qual os dois tipos de distância são considerados através de uma ponderação que leva em conta o número de variáveis em cada caso, como segue:

$$
d_{c}(A, B)=\frac{n_{e} \cdot d_{e}(A, B)+n_{b} \cdot d_{b}(A, B)}{n_{e}+n_{b}}
$$

na qual:

$$
\begin{array}{ll}
\mathrm{d}_{\mathrm{c}}(\mathrm{A}, \mathrm{B}) & \text { - coeficiente combinado de semelhança de Ramesburg ou } \\
& \text { distância competitiva entre a empresa A (referência) e a } \\
& \text { empresa B (empresa em estudo) } \\
\mathrm{n}_{\mathrm{e}} & \text { - número de variáveis quantitativas utilizadas; } \\
\mathrm{d}_{\mathrm{e}}(\mathrm{A}, \mathrm{B}) & \text { - distância euclideana entre a empresa A (referência) e a } \\
& \text { empresa B (empresa em estudo); } \\
\mathrm{n}_{\mathrm{b}} & \text { - número de variáveis dicotômicas utilizadas; } \\
\mathrm{d}_{\mathrm{b}}(\mathrm{A}, \mathrm{B}) & \text { - distância binária de Sokal entre a empresa A (referência) e } \\
& \text { a empresa B (empresa em estudo); }
\end{array}
$$

No caso específico do exemplo considerado neste trabalho, como há 6 variáveis quantitativas $\left(n_{e}=6\right)$ e 6 variáveis dicotômicas $\left(n_{b}=6\right)$, pode-se concluir que o coeficiente combinado de semelhança acaba por se tornar uma média aritmética entre a distância euclidiana e a distância binária de Sokal, ou seja:

$$
\text { Relação (7) }
$$

$$
\mathrm{d}_{\mathrm{c}}(\mathrm{A}, \mathrm{B})=\frac{6 \cdot \mathrm{d}_{\mathrm{e}}(\mathrm{A}, \mathrm{B})+6 \cdot \mathrm{d}_{\mathrm{b}}(\mathrm{A}, \mathrm{B})}{\mathrm{C}+6} \mathrm{\textrm {d } _ { \mathrm { e } } ( \mathrm { A } , \mathrm { B } ) + \mathrm { d } _ { \mathrm { b } } ( \mathrm { A } , \mathrm { B } )}
$$

Como se depreende de toda a consideração teórica desenvolvida, esse coeficiente combinado de semelhança, determinado conforme a Relação (7) acima, permite medir a semelhança, ou diferença, entre o perfil competitivo de uma empresa considerada padrão e o perfil competitivo de outra empresa cujo grau de competitividade se procura medir, 
considerando todas as variáveis competitivas propostas (quantitativas e dicotômicas). Por isso, a esse coeficiente combinado de semelhança, propõe-se atribuir a denominação de distância competitiva entre a empresa A (referência) e a empresa B (caso em estudo).

Concluindo e sumarizando, verifica-se que este trabalho propõe basicamente que o grau de competitividade de uma empresa seja estabelecido por meio da determinação da distância competitiva entre a empresa em análise (empresa B) e o referencial estabelecido (empresa A). Esse cálculo será desenvolvido em três etapas, conforme segue:

a) Variáveis quantitativas - determina-se a distância euclidiana simples entre A e B, onde cada variável competitiva de A é comparada com sua equivalente em B, através da Relação (4) mencionada anteriormente.

b) Variáveis dicotômicas - determina-se a distância binária de Sokal entre A e B, conforme a Relação (5) e o procedimento ilustrado no Quadro 2.

c) Determina-se a distância competitiva entra A e B, calculando-se o coeficiente combinado de semelhança de Romesburg, por meio da aplicação da relação (7) anteriormente mencionada.

Verifica-se, como mostrado, que o cálculo da distância competitiva permitirá determinar quão diferente é a empresa analisada em relação à outra tomada como referência. Com relação ao número obtido para medir essa distância - equivalente ao grau de competitividade de uma empresa - deve-se observar que este é um número puro, desprovido de unidade dimensional, em razão do cálculo ser feito utilizando-se variáveis padronizadas.

Por outro lado, se a empresa em estudo tiver um perfil competitivo idêntico ao da empresa tomada como padrão, a distância competitiva entre elas será nula. De maneira análoga, entre duas empresas distintas comparadas a um mesmo referencial, será mais competitiva a que possuir a menor distância competitiva calculada nos moldes aqui propostos.

\section{UM EXEMPLO ILUSTRATIVO}

Para poder ilustrar a aplicação dos conceitos aqui propostos a situações do mundo real, assuma-se o seguinte exemplo no qual consideram-se três empresas de autopeças pertencentes ao segmento automotivo brasileiro. 
A empresa "P" é considerada o padrão referencial para o segmento de autopeças brasileiro, pois se trata de uma das empresas mais competitivas do ramo industrial no qual ela atua (NETZ, 1999). Servindo o mesmo mercado, há duas outras empresas "A" e "B", cujo grau de competitividade se deseja medir. Assuma-se para os perfis competitivos das três empresas o conjunto de variáveis competitivas mostrado no Quadro 3 abaixo (lembrando que nas variáveis qualitativas a designação 1 significa que a empresa possui o atributo avaliado e a designação 0 indica que ela não o possui).

Deve-se lembrar que os perfis competitivos das empresas "P", "A" e "B" são formados pelo conjunto dos valores das variáveis selecionadas assumidas para cada uma da empresas, como já descrito anteriormente. Assim, com base nessas informações, como avaliar o grau de competitividade das empresas “A” e "B" em relação à empresa padrão "P”?

Quadro 3. O perfil competitivo de três empresas: um exemplo.

\begin{tabular}{|c|c|c|c|}
\hline \multicolumn{4}{|c|}{ Variáveis Quantitativas } \\
\hline Variáveis & “P”' & "A" & "B" \\
\hline Rentabilidade do Patrimônio & $16,6 \%$ & $-24,2 \%$ & $40,2 \%$ \\
\hline Liquidez Corrente & 0,982 & 1,005 & 0,568 \\
\hline Liquidez Geral & 0,710 & 0,681 & 0,394 \\
\hline Endividamento Geral & 0,707 & 0,755 & 0,889 \\
\hline Crescimento de Vendas & $19,1 \%$ & $-12,1 \%$ & $27,6 \%$ \\
\hline Participação de Mercado & $9,4 \%$ & $0,4 \%$ & $1,4 \%$ \\
\hline \multicolumn{4}{|c|}{ Variáveis Qualitativas } \\
\hline Variáveis & 'P'” & "A" & "B" \\
\hline Certificação ISO 9000 & 1 & 1 & 1 \\
\hline Técnicas JIT & 1 & 0 & 0 \\
\hline 5 Princípios de Recursos Humanos & 1 & 0 & 1 \\
\hline Sistema de gestão ambiental & 1 & 0 & 0 \\
\hline Participação no com. internacional & 1 & 0 & 0 \\
\hline Site na Internet & 1 & 0 & 0 \\
\hline
\end{tabular}

Fonte: Elaborado pelo autor. 
Aplicando o desenvolvimento teórico apresentado anteriormente e calculando-se as relações matemáticas sugeridas para as varáveis competitivas em sua forma padronizada, é possível calcular a distância competitiva entre as empresas "A", "B" e o padrão referencial "P", da seguinte forma:

\section{Empresa A}

Varáveis Quantitativas $\quad \rightarrow \mathrm{d}_{\mathrm{e}}(\mathrm{P}, \mathrm{A})=3,915 \rightarrow$ distância euclidiana entre $\mathrm{P}$ e A

Variáveis Dicotômicas $\quad \rightarrow \mathrm{d}_{\mathrm{b}}(\mathrm{P}, \mathrm{A})=0,912 \rightarrow$ distância binária entre $\mathrm{P}$ e A

Variáveis Combinadas $\quad \rightarrow \mathrm{d}_{\mathrm{c}}(\mathrm{P}, \mathrm{A})=2,41 \rightarrow$ distância competitiva entre $\mathrm{P}$ e A

Sabe-se assim, que a distância competitiva entre a empresa "A" e a empresa padrão, é de 2,41. Qual o significado desse número? Em primeiro lugar, é preciso ressaltar que, como o cálculo da distância competitiva é realizado através da utilização de variáveis padronizadas, ela é um número puro, isto é, desprovido de unidade dimensional. Por outro lado, para que a empresa "A" tivesse um perfil competitivo idêntico ao do seu referencial (P), a distância calculada deveria ser zero. Dessa forma, quanto mais próxima de zero for uma distância competitiva, mais semelhante será o perfil competitivo da empresa analisada em relação ao padrão adotado. Do mesmo modo, comparando-se várias empresas em relação a um mesmo referencial, a que tiver menor distância calculada terá uma posição competitiva mais vantajosa em relação a todas as demais.

\section{Empresa B}

Varáveis Quantitativas $\quad \rightarrow \mathrm{d}_{\mathrm{e}}(\mathrm{P}, \mathrm{B})=2,897 \rightarrow$ distância euclidiana entre P e B

Variáveis Dicotômicas $\quad \rightarrow \mathrm{d}_{\mathrm{b}}(\mathrm{P}, \mathrm{B})=0,816 \rightarrow$ distância binária entre $\mathrm{P}$ e B

Variáveis Combinadas $\quad \rightarrow \mathrm{d}_{\mathrm{c}}(\mathrm{P}, \mathrm{B})=1,86 \rightarrow$ distância competitiva entre $\mathrm{P}$ e B

A distância competitiva entre a empresa "B" e a empresa padrão $(1,86)$ é menor do que a anteriormente apurada entre "A" e a empresa padrão $(2,41)$. Isso demonstra ser o perfil competitivo de "B" mais próximo do padrão do que o perfil competitivo da empresa "A", o que permite concluir que a empresa "B" é mais competitiva que a empresa "A", pelos critérios de avaliação aqui propostos. 


\section{CONSIDERAÇÕES FINAIS}

Como se observa pelo desenvolvimento teórico aqui proposto, o conceito de distância competitiva parece adequado para quantificar o grau de competitividade de uma empresa, como se procurou demonstrar ao longo deste trabalho. Por meio da utilização da proposta conceitual aqui apresentada uma empresa poderá também verificar se ela é mais ou menos competitiva se comparada com as suas concorrentes, permitindo inclusive desenvolver ações para a melhoria de sua competitividade (identificando medidas que melhorem os valores de suas variáveis competitivas). No entanto, é importante ressaltar que a competitividade empresarial é um fenômeno bastante complexo, possibilitando análises sob uma extensa gama de enfoques. Assim, ainda que a distância competitiva mostre-se adequada para medi-la, reside na definição das variáveis competitivas a serem selecionadas o verdadeiro desafio a ser superado para uma eficiente medição daquela grandeza.

\section{REFERÊNCIAS}

BUSSAB, W.O., MIAZAKI, E.S. \& ANDRADE, D.F. Introdução à análise de agrupamentos - $9^{\circ}$ Simpósio Brasileiro de Probabilidade e Estatística. São Paulo: Instituto de Matemática e Estatística - USP, 1990.

CASTELLS, Manuel. A sociedade em rede. São Paulo: Paz e Terra, 2000.

COSTA NETO, Pedro Luiz de Oliveira. Estatística. São Paulo: Edgar Blücher, 1997.

DANIELS, J.L. \& DANIELS, N.C. Global vision. New York: McGraw-Hill, 1994.

FERREIRA, Aurélio Buarque de Holanda. Novo aurélio século XXI. Rio de Janeiro: Nova Fronteira, 1999.

HAMEL, Gary \& PRAHALAD, C.K. Competindo pelo futuro. Rio de Janeiro: Campus, 1995.

KOTLER, P. \& ARMSTRONG, G. Principles of marketing. $10^{\text {th }}$ ed. Englewood Cliffs: Prentice-Hall, 2002.

LUCATO, Wagner C. A medida do perfil competitivo de uma empresa: uma proposta conceitual. Anais, VI SemeAD, São Paulo, Faculdade de Economia, Administração e Contabilidade da Universidade de São Paulo, 2003 (CD-ROM).

NETZ, Clayton. As melhores empresas privadas. Exame. São Paulo, Junho 1999, p.76 - 95. 
PFEFFER, Jeffrey. Competitive advantage through people. Boston: Harvard Business School Press, 1996.

PORTER, M. E. Vantagem competitiva. Rio de Janeiro: Campus, 1989.

\& LINDE, C. Green and competitive: ending the stalemate. Harvard Business Review. Sep. / Oct 1995, p.120 - 135.

ROSS, S.A.; WESTERFIELD, R.W. \& JORDAN, B.D. Princípios de administração financeira. 2. ed. São Paulo: Atlas, 2002.

SLACK, N.; CHAMBERS, S. \& JOHNSTON, R. Administração da Produção. 2.ed. São Paulo: Atlas, 2002. 